\title{
Childhood spinal muscular atrophy and stem cell research: Is cellular replacement therapy the answer? (Review)
}

\author{
EMMA O'HARE and PHILIP J. YOUNG \\ Clinical Neurobiology, Institute of Biomedical and Clinical Science, Peninsula Medical School, \\ St. Luke's Campus, Exeter, EX1 2LU, UK
}

Received September 3, 2008; Accepted October 22, 2008

DOI: 10.3892/mmr_00000052

\begin{abstract}
Childhood spinal muscular atrophy (SMA) is an autosomal disorder caused by mutations in the survival motor neuron (SMN) gene. Although SMN is a ubiquitously expressed protein, mutations in SMN specifically cause the targeted deterioration of $\alpha$-motor neurones of the spinal cord. This results in the progressive wasting of the voluntary muscles of the limbs and trunk. Although there is currently no treatment or cure for SMA, several lines of investigation are underway. These include a) screens for small compounds that modulate disease phenotype, b) gene therapy and c) stem cell therapy. In this review, we discuss the current state of stem cell research, concentrating on recent advances and highlighting areas where more research is needed. We also discuss the implications of this research for SMA.
\end{abstract}

\section{Contents}

1. Introduction

2. Overview of stem cells

3. Stem cell-derived motor neurone generation and its therapeutic potential

4. Discussion: prospects and considerations for future use of cellular transplantation in SMA

\section{Introduction}

Childhood spinal muscular atrophy (SMA) is a common recessive autosomal disorder characterised by deterioration of $\alpha$-motor neurones in the spinal cord and brain stem nuclei, with consequent muscle wasting and paralysis of the limbs and trunk (1). The disease is caused by deletion or mutations of the telomeric copy (SMN1) of the survival motor neuron (SMN)

Correspondence to: Dr Philip J. Young, Clinical Neurobiology, Institute of Biomedical and Clinical Science, Peninsula Medical School, St. Luke's Campus, Exeter, EX1 2LU, UK

E-mail: philip.young@pms.ac.uk

Key words: spinal muscular atrophy, stem cell research, replacement therapy gene located on chromosome $5 q 13$, resulting in a marked reduction in SMN protein (1). Absolute SMN protein levels are ultimately governed by the number of remaining centromeric copies (SMN2) of the SMN gene, which is able to produce only a fraction of identical full-length transcripts in comparison to SMN1 (1-3). Increased copy numbers of SMN2 and the subsequent increased production of full-length SMN protein manifest as progressively milder forms of the disease (1-3).

The clinical features of SMA result from skeletal muscle denervation (4). The disorder can be subdivided into three groups according to age of onset, the severity of symptoms (assessed by the achievement of motor milestones) and age at death (1,2,5-8). SMA type I (Werdnig-Hoffmann disease) is the most severe form, typically describing infants diagnosed before six months of age experiencing such profound weakness as to render them incapable of ever achieving an independent sitting posture, with death by age two $(1,3)$. SMA type II is intermediate in severity, with an average age of onset earlier than 18 months $(1,3)$. Type II infants can usually survive beyond the first two years and sit independently; however, they are generally too weak to ever walk unaided. Individuals with SMA type III (Kugelberg-Welander disease), the mildest form of the disorder, experience weakness after 18 months but are able to maintain a standing posture and walk $(1,3)$. These children have a normal life expectancy $(1,3)$.

In spite of worldwide efforts, there is as yet no cure for SMA (9-11). Principal approaches to finding an effective treatment or indeed a cure have been aimed at either manipulating the genetic material implicated in the pathophysiology of SMA, or at restoring the lost or damaged motor neurones via cellular replacement (9-11).

This study focuses on stem cell therapy as a potential treatment for SMA, reviewing relevant studies as well as considering the major issues associated with the implementation of this therapy in clinical practice.

\section{Overview of stem cells}

Stem cells are immature precursor cells that are able to selfrenew and to create multiple mature cell types, depending on their origin (12). This capacity for differentiation can be further described as totipotent, pluripotent or unipotent, depending on whether the cells are able to produce any cell type, several cell types, or only one cell type, respectively (12). Pluripotent embryonic stem cells are obtained from the 
inner-cell mass of cultured embryos at the blastocyst stage of development, and are the underpinning of the majority of research conducted on cellular therapy (13). Although some adult tissues do harbour stem cells, they tend to be fewer in number, proliferate less, and are less potent compared to embryonic stem cells (13).

\section{Stem cell-derived motor neurone generation and its therapeutic potential}

The fundamental rationale for the use of stem cells in degenerative disorders such as SMA is that these progenitors can potentially differentiate into mature cell lines under the influence of developmental signals appropriate to that cell type (12).

The generation of motor neurones, the affected cell type in SMA, has been achieved in the lab by exposing mouse embryonic stem cells to retinoic acid (RA) and nerve growth factor (NGF). These together target Sonic hedgehog (Shh), a developmental morphogen, and convert spinal progenitors to motor neurones $(14,15)$. In one well-cited study, when treated cells were injected into the spinal cord of an embryonic chick, they migrated to the proper location on the ventral horn. Some were even able to extend into the periphery and form neuromuscular junctions on target muscle fibres (14). This research strongly suggests that embryonic stem cells could be made to replicate normal developmental pathways for the effective generation of motor neurones.

To be considered a feasible therapy, lab-generated motor neurones must firstly be able to survive within the spinal cord once implanted, and secondly to extend axons through proximate white matter into the periphery to re-innervate appropriate target tissue (16-18).

Kerr et al, in 2003, were unable to establish functional neuromuscular junctions in the host nervous system (19). In this study, rats were infected with Neuroadapted Sindbis virus, an RNA virus known to induce hind leg paralysis in rodents (19). Once paralysed, the rats were subjected to the transplantation of motor neurones derived from a stem cell source and assessed for restoration of function (19). Notably, the paralysed rats did display some functional recovery, despite the lack of evidence of synapse formation (19). These researchers speculated that the partial recovery was not due to the re-establishment of stem cell-derived motor neurones, but rather to the protective and restorative effect of trophic factors secreted by donor motor neurones on the host cells (19). Indeed, brain-derived neurotrophic factor and transforming growth factor- $\alpha$, two factors known to be implicated in the survival and axonal growth of motor neurones, were found in moderate-to-increased levels in the rats, strongly suggesting that some degree of neurotrophic support had taken place (19).

Other studies have also investigated the potential of cellular therapy to stop motor neurone degeneration and reestablish function in animals with neurodegenerative disease or spinal cord injury.

In 2004, Harper et al studied embryonic stem cell-derived motor neurones in an in vitro environment, as well as after transplantation into paralysed rats (16). They found that motor neurones derived from stem cells were able to grow axons, form neuromuscular junctions and induce muscle contraction in vitro (16). Three months after transplantation into the rats, approximately 3000 of the original 12,000 neurones had survived. Of these, only 75 (approximately 2.4\%) had grown axons through the surrounding white matter of the spinal cord into the periphery, even after treatment with blockers of myelin-mediated axonal inhibition (16). In addition, there was no evidence of neuromuscular junction formation by the transplanted motor axons in vivo and, accordingly, no electrophysical or functional recovery of the paralysed rats (16).

It was not until 2006 that a study by Despande et al confirmed that stem cell-derived motor neurones were able to restore function in paralysed rats through the formation of neuromuscular junctions in vivo (18). As in the other studies, pluripotent stem cells were firstly directed to differentiate into committed motor neurone progenitors by the use of RA and a chemical agonist of Shh (18). To promote axon regeneration once transplanted, other agents were administered to the stem cell-derived motor neurones to stimulate axonal growth and survival, as well as to prevent the inhibitory effects of myelin. These were dibutyryl cyclic adenosine monophosphate (dbcAMP) and rolipram (a phosphodiesterase inhibitor), respectively. In contrast to the other studies, glial cell-derived neurotrophic factor (GDNF), a vigorous stimulator of motor axonal growth, was also applied in order to direct axons distally to form neuromuscular junctions. Initial findings revealed a significant increase in the number of functioning motor neurones in the rats that had received all treatments, compared to control animals in which various stages of the process had been omitted (18). In addition, four months after transplantation, the fully-treated rats demonstrated an improvement in hind limb grip strength of approximately $50 \%$ (18).

\section{Discussion: prospects and considerations for future use of cellular transplantation in SMA}

To date, there is no cure for individuals affected with SMA $(10,11,20)$, yet the US National Institutes of Health have declared SMA to be the genetic condition closest to an effectual treatment or cure $(10,11,20)$. Stem cell therapy, if successful, could theoretically delay onset and advancement of the disease, as well as potentially restoring lost function $(10,11,20)$.

Specific features of SMA make this condition particularly suitable for future cellular replacement therapy techniques. In contrast to other neurodegenerative disorders such as spinal cord injury or stroke, where a multifaceted pathology affects many targets, only the spinal motor neurones are affected in SMA; hence, the target for therapy has been identified $(10,11,20)$. In addition, the environment around the spinal cord in SMA individuals is more favourable for a potential graft $(10,11,20)$. Type I infants would almost certainly have a better supply of blood and growth factors to the area than would be seen in, for instance, spinal cord injury, because these newborns are growing rapidly $(10,11,20)$. Furthermore, the distance travelled by any sprouting axons from the transplanted motor neurones to reach affected muscle would be shorter in SMA than in other neurodegenerative diseases, 
owing to the fact that this pathology primarily affects infants and children $(10,11,20)$. Thus, synapses with host muscle would form sooner, leading to an earlier improvement in function.

Various studies on stem cell therapy have already garnered significant attention for their impact on the treatment of diseases such as Alzheimer's, Parkinson's and type I diabetes mellitus (13). Scientific research on the use of stem cells to help preserve or reinstate vulnerable motor neurones has been progressing rapidly (11); however, there are still considerable obstacles to be overcome and other issues to be addressed before this potential therapy can be of benefit to SMA patients.

Controversy surrounding the use of stem cells, particularly those of embryonic origin, has been well documented (13). Opposition from various religious and political quarters will clearly need to be addressed in order to aid the scientific community in the quest for a viable stem cell therapy (13).

As is evident from the abovementioned studies, rodent models of neurodegenerative diseases such as SMA have given researchers the opportunity to test the potential of cellular transplantation (21). While the results from these studies have been promising, the current challenge involves confirming these findings in models using larger animals to determine whether neurones can reconnect over extended distances, as well as to ascertain the safety of the treatments. With this in mind, the pig (Sus scrofa) SMN gene has recently be identified and characterised (22).

In addition, since knowledge of the developmental biology related to inducing the motor neurone lineage is key to the use of human embryonic stem cells, it is essential that this process be defined and perfected before implementation, as has been done in mouse cells $(10,18)$.

Other targets for stem cell therapy in SMA must also be contemplated. Some authorities suggest that stem cells could additionally be used to prevent muscle atrophy or replace lost muscle (23). Arguments for this approach use the reasoning that SMA is a disease of both muscle and nerve, so to attempt the recovery of one without the concurrent recovery of the other is not beneficial in the long term (23).

To conclude, it appears that in the near future cellular replacement therapy could indeed be the answer for patients with SMA. It is also evident that, like many other new or innovative potential therapies, the approach is still far from being implemented in a clinical setting. It is imperative that more research be conducted, both in the areas discussed above and on other therapeutic strategies, to provide hope to the sufferers of this common genetic disorder and their families.

\section{Acknowledgements}

The authors would like to thank Drs E.C. Young and Robert Morse for their comments and assistance. P.J.Y. is sponsored by the Vandervell Foundation, Fight SMA and the SMA Trust.

\section{References}

1. Lefebvre S, Burglen L, Reboullet S, et al: Identification and characterization of a spinal muscular atrophy-determining gene. Cell 80: 155-165, 1995.

2. Lefebvre S, Burglen L, Frézal J, Munnich A and Melki J: The role of the SMN gene in proximal spinal muscular atrophy. Hum Mol Genet 7: 1531-1536, 1998.

3. Lefebvre S, Burlet P, Liu Q, et al: Correlation between severity and SMN protein level in spinal muscular atrophy. Nat Genet 16: 265-269, 1997.

4. Nicole S, Diaz CC, Frugier T and Melki J: Spinal muscular atrophy: recent advances and future prospects. Muscle Nerve 26: 4-13, 2002.

5. Wang CH, Xu J, Carter TA, et al: Characterization of survival motor neuron (SMNT) gene deletions in asymptomatic carriers of spinal muscular atrophy. Hum Mol Genet 5: 359-365, 1996.

6. Campbell L, Potter A, Ignatius J, Dubowitz V and Davies K: Genomic variation and gene conversion in spinal muscular atrophy: implications for disease process and clinical phenotype. Am J Hum Genet 61: 40-50, 1997.

7. Coovert DD, Le TT, McAndrew PE, et al: The survival motor neuron protein in spinal muscular atrophy. Hum Mol Genet 6: 1205-1214, 1997.

8. Taylor JE, Thomas NH, Lewis CM, et al: Correlation of SMNt and SMNc gene copy number with age of onset and survival in spinal muscular atrophy. Eur J Hum Genet 6: 467-474, 1998.

9. Heier CR, Gogliotti RG and DiDonato CJ: SMN transcript stability: could modulation of messenger RNA degradation provide a novel therapy for spinal muscular atrophy? J Child Neurol 22: 1013-1018, 2007.

10. Kostova FV, Williams VC, Heemskerk J, et al: Spinal muscular atrophy: classification, diagnosis, management, pathogenesis, and future research directions. J Child Neurol 22: 926-945, 2007.

11. Swoboda KJ, Kissel JT, Crawford TO, et al: Perspectives on clinical trials in spinal muscular atrophy. J Child Neurol 22: 957-966, 2007.

12. Nayak MS, Kim YS, Goldman M, Keirstead HS and Kerr DA: Cellular therapies in motor neuron diseases. Biochim Biophys Acta 1762: 1128-1138, 2006.

13. Fischbach GD and Fischbach RL: Stem cells: science, policy, and ethics. J Clin Invest 114: 1364-1370, 2004.

14. Wichterle H, Lieberam I, Porter JA and Jessell TM: Directed differentiation of embryonic stem cells into motor neurons. Cell 110: 385-397, 2002.

15. So PL, Yip PK, Bunting S, et al: Interactions between retinoic acid, nerve growth factor and sonic hedgehog signalling pathways in neurite outgrowth. Dev Biol 298: 167-175, 2006.

16. Harper JM, Krishnan C, Darman JS, et al: Axonal growth of embryonic stem cell-derived motoneurons in vitro and in motoneuron-injured adult rats. Proc Natl Acad Sci USA 101: 7123-7128, 2004

17. Darman J, Backovic S, Dike S, et al: Viral-induced spinal motor neuron death is non-cell-autonomous and involves glutamate excitotoxicity. J Neurosci 24: 7566-7575, 2004.

18. Deshpande DM, Kim YS, Martinez T, et al: Recovery from paralysis in adult rats using embryonic stem cells. Ann Neurol 60: 32-44, 2006.

19. Kerr DA, Lladó J, Shamblott MJ, et al: Human embryonic germ cell derivatives facilitate motor recovery of rats with diffuse motor neuron injury. J Neurosci 23: 5131-5140, 2003.

20. Nadeau A, D'Anjou G, Debray G, Robitaille Y, Simard LR and Vanasse M: A newborn with spinal muscular atrophy type 0 presenting with a clinicopathological picture suggestive of myotubular myopathy. J Child Neurol 22: 1301-1304, 2007.

21. Frugier T, Nicole S, Cifuentes-Diaz C and Melki J: The molecular bases of spinal muscular atrophy. Curr Opin Genet Dev 12: 294-298, 2002.

22. Lorson MA, Spate LD, Prather RS and Lorson CL: Identification and characterization of the porcine (Sus scrofa) survival motor neuron (SMN1) gene: An animal model for therapeutic studies. Dev Dyn 237: 2268-2278, 2008.

23. Monani UR: Spinal muscular atrophy: a deficiency in a ubiquitous protein; a motor neuron-specific disease. Neuron 48: 885-896, 2005. 
